\title{
An atypical case of acute kidney injury: Questions
}

\author{
Caroline Weisser $^{1}$ - Janusz Feber ${ }^{1,2} \cdot$ Anne Tsampalieros ${ }^{1,2} \cdot$ Christoph Licht $^{3,4}$
}

Received: 6 March 2015 /Revised: 9 March 2015 / Accepted: 10 March 2015 / Published online: 15 April 2015

(C) IPNA 2015

Keywords Acute kidney injury $\cdot$ Hypocomplementemia . S. pneumoniae infection · Atypical $\cdot$ Renal function analysis

\section{Case}

A previously healthy 9-month-old boy presented to the hospital with a 10-day history of decreased energy, feeding difficulties, maculopapular rash, vomiting, and fever. He had been diagnosed with pneumonia and started on oral amoxicillin 2 days before hospital admission. On physical examination, he had a rectal temperature of $38.5^{\circ} \mathrm{C}$, a pulse of 134 beats per minute, and a blood pressure of 118/69 mmHg. He had mild intercostal retractions with abdominal breathing and decreased air entry at the left base. He had mild periorbital edema and a maculopapular rash on the trunk and upper extremities. He was started on intravenous (IV) fluids because of clinical signs of dehydration (estimated body weight loss of 5-10\%). A chest X-ray confirmed a left lower lobe pneumonia with moderate effusion, his antibiotics were changed to IV cefuroxime and vancomycin, and he was admitted to the hospital. Overnight he developed non-bloody diarrhea.

While his urine output was within normal limits, his initial renal function analysis revealed a slightly elevated serum creatinine $(\mathrm{SCr})$ of $48 \mu \mathrm{mol} / \mathrm{L}$ and a blood urea of $12.1 \mathrm{mmol} / \mathrm{L}$,

The answers to these questions can be found at http://dx.doi.org/10.1007/ s00467-015-3092-8

Anne Tsampalieros

atsampalieros@cheo.on.ca

University of Ottawa, Ottawa, ON, Canada

2 Division of Nephrology, Children's Hospital of Eastern Ontario, 401 Smyth Road, Ottawa, ON K1H 8L1, Canada

3 The Hospital for Sick Children, Toronto, Ontario, Canada

4 University of Toronto, Toronto, Ontario, Canada likely due to dehydration. The white cell and platelet counts were normal at $4.83 \times 10^{9}$ and $302 \times 10^{9} / \mathrm{L}$, respectively; hemoglobin was decreased to $105 \mathrm{~g} / \mathrm{L}$. Urinalysis was negative for protein, blood, and nitrites. A repeat blood test performed $24 \mathrm{~h}$ later showed a rise in $\mathrm{SCr}$ to $60 \mu \mathrm{mol} / \mathrm{L}$ and in blood urea to $17.6 \mathrm{mmol} / \mathrm{L}$. Subsequently, his hemoglobin dropped to $73 \mathrm{~g} / \mathrm{L}$ and his platelets dropped to $19 \times 10^{9} /$ L. Further work-up revealed an elevated lactate dehydrogenase at 20,494 (normal values 500-900) U/L, a negative Coombs test, and schistocytes on blood smear. Blood cultures and urine cultures remained sterile; $\mathrm{C} 3$ and $\mathrm{C} 4$ complement levels were still pending at this time.

The patient was subsequently admitted to the intensive care unit where his urine output started to drop. He became completely anuric on Day 1 after admission, and his $\mathrm{SCr}$ and blood urea progressively increased over time, reaching a maximum of 182 and $29.4 \mathrm{mmol} / \mathrm{L}$, respectively. Progressive uremia, ongoing anuria, and fluid overload prompted the initiation of hemodialysis via a central line on Day 2 after admission. Daily hemodialysis was required for 2 weeks due to severe anuria which was accompanied by elevated SCr. During that period, the antibiotic regimen was changed to IV ceftriaxone. He required three packed red cell transfusions and two platelet transfusions due to ongoing anemia and thrombocytopenia. In addition, amlodipine was initiated to control his hypertension. The $\mathrm{C} 3$ and $\mathrm{C} 4$ complement levels were low on two occasions, with $\mathrm{C} 3$ at 0.57 and 0.49 (normal values $0.7-1.8$ ) $\mathrm{g} / \mathrm{L}$ and $\mathrm{C} 4$ at 0.06 and 0.06 (normal values $0.15-0.45) \mathrm{g} / \mathrm{L}$, respectively.

\section{Questions}

1. What is the diagnosis?

2. What other tests could be performed to help confirm diagnosis?

3. How should the patient be treated? 